EUROPA

\title{
Lobbying für das Soziale
}

\author{
Michael Himmer
}

Eine erfolgreiche und wirksame Interessensvertretung sozialer Anliegen verlangt neue Unternehmensstrategien der Sozialwirtschaft und umfasst neben der europäischen Vernetzung vor allem ein regionales und nationales Soziallobbying.

Über europäische Entwicklungen informierte Organisationen erkennen früher die künftigen Rahmenbedingungen und wichtige soziale Entwicklungen zur strategischen Steuerung des Unternehmens, starten früher mit Projektkonzepten und sie haben bei Bedarf rascher Verbündete. Das sind Kernelemente für die Lebensfähigkeit auch von Sozialorganisationen und ihrem Auftrag, Menschen in Not zu helfen.

Deshalb muss europäische Netzwerkarbeit und Lobbying professionalisiert werden. Lobbying umfasst neben allen Formen des Direktkontaktes und der persönlichen Kommunikation mit relevanten Entscheidungsträgern vor allem Formen des indirekten Lobbyings, also der Kooperation mit Dritten, um Entscheidungen zu beeinflussen.

Wichtigstes Instrument ist ohne Zweifel die Vernetzung mit anderen Organisationen in europäischen Dachverbänden. Über solche Netzwerke werden direkte Informationskanäle zu Entscheidungsträgern in Brüssel aufgebaut. Rechtliche, strategische und finanzielle Initiativen auf europäischer Ebene können so frühzeitig erkannt werden und aktiv in die Unternehmensplanung einfließen.

Europäische Initiativen, Studien und Trends zeigen eindrücklich, dass sich Europa in der Frage der Ausrichtung der Sozialwirtschaft und sozialer Dienstleistungen einer neuen Verantwortung bewusst wird; auch wenn die Frage nach einer Definition der Sozialwirtschaft eher akademische Züge trägt. Das Europäische Parlament hat die Sozialwirtschaft als »Grundpfeiler des Europäischen Sozialmodells« bezeichnet und eigene, neue Erfolgskriterien gefordert. Im Gegensatz dazu sieht der Europäische Wirtschafts- und Sozialausschuss die Sozialwirtschaft in einer doppelten Funktion und meint: »... sie erfüllt wesentliche Aufgaben, insbesondere im Pflege- und Fürsorgesektor, während sie gleichzeitig Bürgern, die in der normalen Arbeitswelt nur schwer Fuß fassen können, wie Menschen mit Behinderungen, Arbeit bietet.« Damit reduziert der Europäische Wirtschafts- und Sozialausschuss die Sozialwirtschaft auf einen »Arbeitsmarkt für nicht-integrierbare Menschen«.

Die angekündigte Europäische Richtlinie zu sozialen Diensten oder das Anhörungsverfahren zur sozialen Wirklichkeit der Europäischen Kommission sind weitere Schritte in der Neuorientierung der »Europäischen Sozialwirtschaft«.

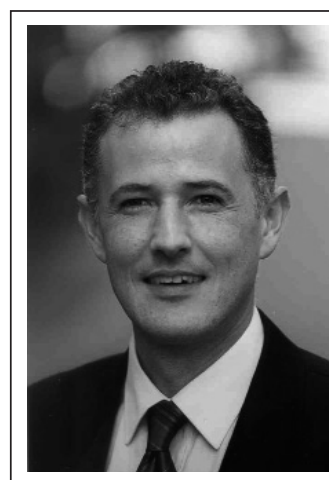

Dr. Michael Himmer wurde 1964 in Vorarlberg geboren. Das Rechtsstudium und die berufliche Laufbahn in der Verwaltung, in der Unternehmensberatung, in der Sozialwirtschaft sowie in nationalen und europäischen Netzwerken haben ihn zum Thema der europäischen Sozialpolitik geführt. Heute arbeitet er als Berater für Regierungen, Sozialunternehmen und Netzwerke und als Lehrbeauftragter mit dem Schwerpunkt Europäische Sozialpolitik. Internet http://www.eu-sozialpolitik.com

Im Sozialbereich sind effiziente Netzwerke rar und transparente, zielorientierte Zusammenarbeit noch selten. Die wichtigsten europäischen Netzwerke sind in der »European social platform « (www.socialplatform.org) vereint und diese versuchen, europäische Entscheidungsprozesse zu beeinflussen. Wichtige Aufgaben bestehen darin, der jeweiligen Ratspräsidentschaft am Beginn einen Fragenkatalog zu sozialen Entwicklungen und geplanten Initiativen vorzulegen, begleitend konsequent zu intervenieren und nach Ablauf eine Evaluierung der Sozialpolitik vorzunehmen. Aktive Netzwerke sind zum Beispiel EASPD (European Association of service providers for people with disabilities, www.easpd.eu), die Europäische Armutskonferenz (www.eapn.org) oder SOLIDAR (www.solidar.org).

\section{Regionales und nationales Soziallobbying}

»Multiplikatoren-Management « zielt nicht unmittelbar auf die Vernetzung, sondern auf die Information und Beeinflussung von Schlüsselspielern. Diese sind nicht nur die Entscheidungsträger selbst, sondern oftmals taktisch agierende »Berater « und »Konsulenten « und Mitglieder von Beratungsgremien auf europäischer, nationaler und regionaler Ebene. So haben die Mitglieder des Ausschusses der Regionen Einfluss auf die Meinungsbildung in der Europäischen Union, die Vertreterinnen und Vertreter kommen aus den Bundesländern und sind Politiker aus ihrem Umfeld. Das Gleiche gilt für Mitglieder des Europäische Wirtschafts- und Sozialausschusses und anderer Gremien. Über Kontakte mit diesen Personen können regionale Interessen wirksam bis nach Brüssel durchdringen. 


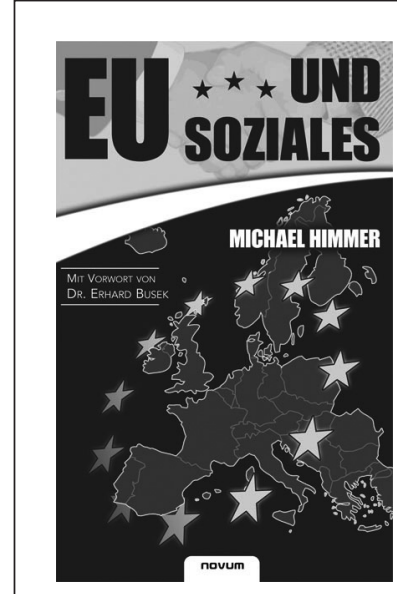

Mit dem Buch »EU und Soziales « bietet Michael Himmer einen Einstieg in die vielfältigen Verbindungen zwischen europäischer, nationaler und regionaler Sozialpolitik. Die Meilensteine der europäischen Sozialpolitik zeigen: Die Weichen für ein gemeinsames Modell sind gestellt. Der Autor macht die Fakten sichtbar: Zuständigkeiten und rechtliche Rahmen-

bedingungen, Programme und Arbeitsprinzipien, konkrete sozialpolitische Strategien. Und er weist auf informelle Positionen, Erwartungen und Rollen hin, macht Entwicklungen und Trends verständlich und zeigt die Herausforderungen für soziale Entscheidungsträger.

Michael Himmer: EU und Soziales. Verlag novum, Neckenmarkt (Österreich) 2006. 220 Seiten. 15,90 Euro. ISBN 978-3-902536-01-3.

Auch kurzfristige Interessenskoalitionen können als Instrument der Beeinflussung von Rahmenbedingungen nützlich sein. Gerade in einzelnen Initiativen oder Programmen der Europäischen Union, in denen kurz- oder mittelfristig erhebliche Dynamik entstehen kann, sind themenbezogene Kooperationen mitunter hilfreich. So kann eine wirksame, wenn auch unterschiedlich motivierte Zusammenarbeit zwischen Behinderteneinrichtungen und der Bauwirtschaft für das menschengerechte Bauen sinnvoll und Erfolg versprechend sein.

\section{Die neue Herausforderung}

Ein ganz wesentliches Element für erfolgreiches Soziallobbying ist die Assistenzleistung für die Verwaltung und die Politik. Recherchen, Positionspapiere, vorbereitende Stellungnahmen oder Textentwürfe zu Themen werden vorgelegt. Diese Tätigkeit verlangt eine neue Einstellung sozialer Organisationen vom »Anwalt sozialer Not « zum »Profiberater für soziale Entwicklungen«. Die Assistenzleistung umfasst auch eine ausgewiesene Expertise in der Berechnung von Kosten und Nutzen (Szenarienentwicklung, Benchmarking von Sozialkosten, Kosten unterlassener sozialer Dienstleistungen etc.), ohne den sozialpolitischen Auftrag zu vernachlässigen. Modernes Lobbying baut auf dem gemeinsamen Suchen nach den besten Lösungen auf. Vor allem dann, wenn andere Formen des Lobbyings für soziale Anbieter zu teuer sind.

Zwischen europäischen Institutionen und den Mitgliedstaaten soll die offene Methode der Koordinierung zur besseren Meinungsbildung beitragen. Auf nationaler und regionaler Ebene sind leider nur wenige, griffige und transparente Verfahren mit den Organisationen der Zivilgesellschaft sichtbar. Nationale und regionale Regierungen müssen sich einlassen auf eine neue Form der Koope-

\section{Kompetent durch Information}

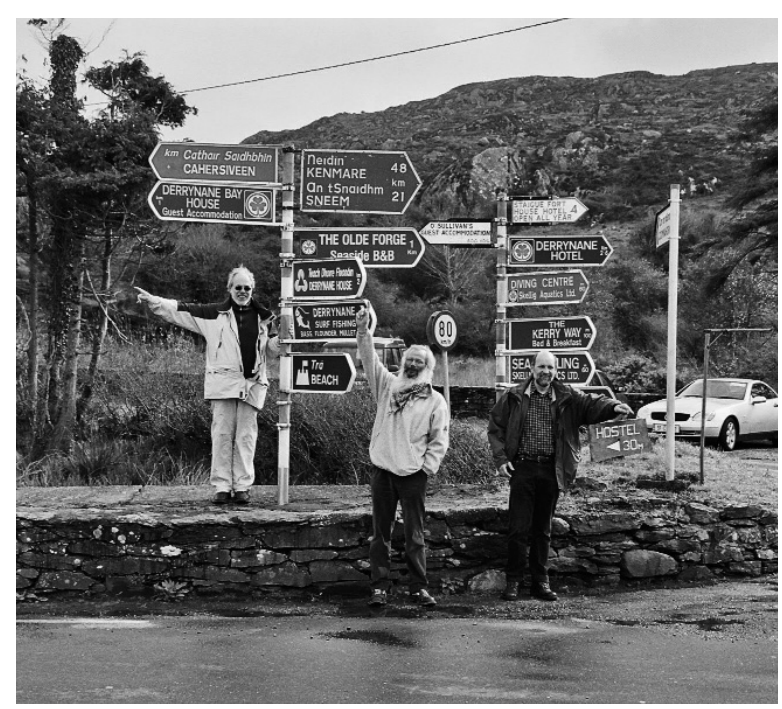

Es ist Information, die dem Sozial- und Gesundheitsbereich fehlt.

Soziale Dienstleistungsunternehmen entwickeln aus ihrem Arbeitsauftrag heraus eine überragende Ausrichtung konkret am Menschen in Not. Mitarbeiter konzentrieren sich ausschließlich auf die Bedürfnisse des Klienten. So wertvoll diese Einstellung ist, so gefährlich ist die Ignoranz vor allgemein politischen und europäischen Entwicklungen. Es ist die Information zur Steuerung und Gestaltung des eigenen politischen und betrieblichen Umfeldes. Wer zu lange vor den entscheidenden Entwicklungen die Augen verschließt, den werden diese überholen. Wenn Sozialpolitik europäisch wird, dann müssen europäische Entwicklungen in allen Bereichen der sozialen Arbeit stärker berücksichtigt werden.

Es sind die Sozialorganisationen selbst, die sich darauf einlassen müssen. Wer glaubt, dass Beobachten als Strategie der Unternehmensführung ausreicht, erweist der Organisation und den eigenen Klienten keinen Dienst. Die Kompetenz nimmt mit dem Grad der aktuellen Information zu. Vor allem im Verbund können die wichtigen Informationen auch aus dem Übermaß der immer weiter zunehmenden Papierflut gefiltert werden. Informierte Organisationen erkennen früher die künftigen Rahmenbedingungen zur strategischen Steuerung des Unternehmens, sie starten früher mit Projektplanungen und sie haben bei Bedarf rascher Verbündete. Das sind Kernelemente für die Lebensfähigkeit auch von Sozialorganisationen und ihrem Auftrag, Menschen in Not zu helfen.

Michael Himmer in seiner kurzen "Einführung zum Thema Europäische Sozialpolitik«. Das 17-seitige Manuskript kann als PDF-Datei von seiner Website kostenlos heruntergeladen werden (http://www.eu-sozialpolitik.com/skriptum_eu_sozialrecht.pdf). 
ration und die Sozialwirtschaft muss in einem neuen Rollenverständnis den zivilen Dialog einfordern. Die Alternative ist sozialer Stillstand!

Das Europäische Sozialmodell, das es (noch) nicht gibt, wird zu einer Kernfrage für den Erfolg der europäischen Idee. Wettbewerb und Wachstum werden als Grundlage für ein neues Europa nicht ausreichen. Der Mensch muss im Zentrum der politischen Diskussion und Aktion stehen, in dem soziale Anliegen und konkrete Notlagen der Menschen aufgegriffen und gelöst werden.
Die Abstimmungen gegen die Europäische Verfassung in Frankreich und den Niederlanden haben gezeigt, dass es keinen Integrationsprozess ohne oder gegen die Bürger in Europa gibt. Für die Sozialwirtschaft mit ihrer überragenden Rolle in der Gesellschaft resultiert daraus eine Fülle von Chancen. Diese Chancen können genützt werden mit einer neuen offensiven Interessensvertretung auf allen Ebenen sozialpolitischer Entscheidungen, wobei die koordinierende Verantwortung der Europäischen Union spürbar zunehmen wird.

\section{Öffentliche Beschaffungspolitik im Wandel}

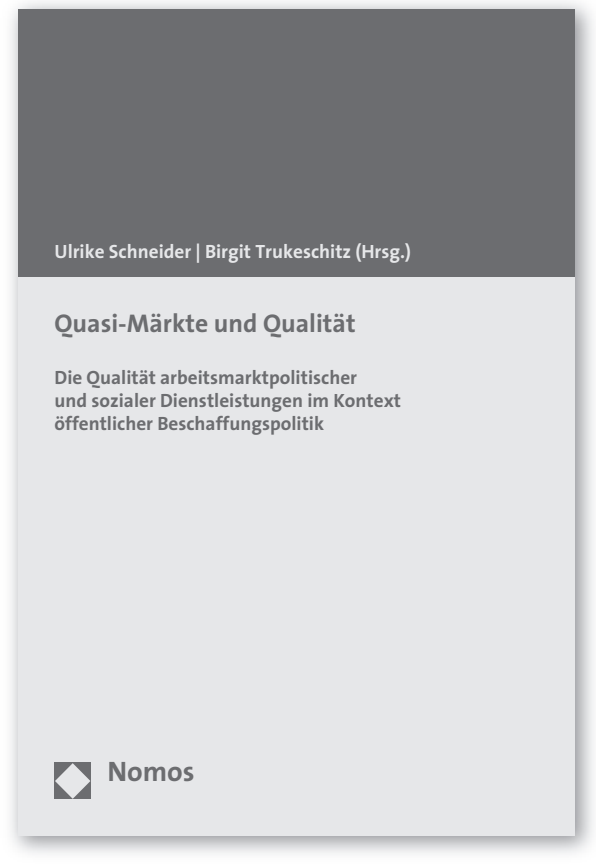
öffentlichen Beschaffung arbeitsmarktpolitischer und sozialer Dienst-
leistungen die Qualität der Angebote von und die Arbeitssituation in Organisationen der Sozialwirtschaft in Österreich beeinflussen.

Die AutorInnen befassen sich mit dem Konzept der Qualität, diskutieren ausgewählte Beschaffungsverfahren und präsentieren Befunde zu den Handlungsspielräumen und Qualitätswirkungen unterschiedlicher öffentlicher Beschaffungsmodi aus ökonomischer, organisationswissenschaftlicher und rechtswissenschaftlicher Sicht.

\begin{abstract}
Quasi-Märkte und Qualität
Die Qualität arbeitsmarktpolitischer und sozialer Dienstleistungen im Kontext öffentlicher Beschaffungspolitik Herausgegeben von Prof. Dr. Ulrike Schneider, Department Volkswirtschaft, Wirtschaftsuniversität Wien; Leitung des Instituts für Sozialpolitik und des Forschungsinstituts für Altersökonomie und Dr. Birgit Trukeschitz, Institut für Sozialpolitik, Wirtschaftsuniversität Wien
\end{abstract} 2007, 310 S., brosch., 24,90€, ISBN 978-3-8329-3073-8

Die öffentliche Beschaffungspolitik im Sozialwesen befindet sich im Wandel. Durch Deregulierung und Öffnung der Märkte, die über die europäische Politik beschleunigt werden, kommen verstärkt marktorientierte Beauftragungen in Bereichen zur Anwendung, die zuvor über Förderungen bzw. auf Basis direkt vergebener Aufträge öffentlich finanziert wurden. Wissenschaftliche Studien zu den Effekten der neuen öffentlichen Beschaffungspolitik im Sozialwesen sind bislang rar. Dort, wo eine erste Bilanz vorhanden ist, liegt der Fokus meist auf der Kostenwirkung wettbewerblicher Beschaffungsverfahren.

Dieses Buch geht der Frage nach, wie unterschiedliche Verfahren der öffentlichen Beschaffung arbeitsmarktpolitischer und sozialer Dienst-
die Arbeitssituation in Organisationen der Sozialwirtschaft in Öster(1) 